\title{
EFFECT OF THREE-BODY INTERACTIONS ON THE FORMATION ENTROPY OF MONOVACANCIES IN COPPER, SILVER AND GOLD
}

\author{
W. MAYSENHÖLDER, R. BAUER and A. SEEGER \\ Max-Planck-Institut für Metallforschung, Institut für Physik, \\ Heisenbergstrasse 1, D 7000 Stuttgart 80, Fed. Rep. Germany \\ and Universität Stuttgart, Institut für Theoretische und Angewandte Physik, \\ Pfaffenwaldring 57, D 7000 Stuttgart 80, Fed. Rep. Germany
}

Received 21 February 1985; revised manuscript received 12 April 1985; accepted for publication 16 April 1985

\begin{abstract}
The vacancy formation entropy in $\mathrm{Cu}, \mathrm{Ag}$ and $\mathrm{Au}$ is estimated from model calculations with explicit treatment of three-body interactions. The three-body interactions cause a rather strong relaxation around the vacancies and therefore lead to lower values for the formation entropy than usual pair-potential calculations.
\end{abstract}

In a recent letter [1] we have reported calculations on point defects in $\mathrm{Cu}, \mathrm{Ag}$ and $\mathrm{Au}$ based on model potentials [2] that allow explicitly for three-body interactions by including a modified Axilrod-Teller potential [3]. In contrast to the common pair-potential approach, the elastic constants $c_{11}, c_{12}, c_{44}$ are reproduced exactly and need no longer be "adjusted" for Cauchy's relation $c_{12}=c_{44}$ to be satisfied. We expect the inclusion of the three-body term to permit a meaningful comparison of $\mathrm{Cu}, \mathrm{Ag}$ and $\mathrm{Au}$.

The properties calculated in ref. [1] include formation energies and formation volumes of mono- and di-vacancies and of four interstitial configurations. .The three-body interactions led to noticeable lattice relaxations around the vacancies: The experimental value of the monovacancy relaxation volume $V_{1 \mathrm{~V}}^{\mathrm{rel}}$ in $\mathrm{Cu},-(0.20 \pm 0.05) \Omega$ [4] ( $\Omega$ is atomic volume), is reproduced, whereas usual pair-potential calculations yield only about $-0.02 \Omega$ (see, e.g., ref. [5]). Encouraged by this result we proceed to calculate the monovacancy formation entropy $S_{1 \mathrm{Y}}^{\mathrm{F}}$.

In the first attempt to calculate $S_{1 \mathrm{~V}}^{\mathrm{F}}$ for $\mathrm{Cu}$, Huntington, Shirn, and Wajda [6] arrived at $1.5 k_{\mathrm{B}}$ assuming Born-Mayer repulsion between the atoms. In 1964 Schottky, Seeger, and Schmid [7] obtained $0.5 k_{\mathrm{B}}$ using a (non-central) force constant model which gives a vacancy relaxation of $-0.13 \Omega$. In both calculations, performed before modern computers were available, the underlying models could not be evaluated with the desirable accuracy. This may be seen from the calculations by Hatcher, Zeller and Dederichs [8], which demonstrated that considerable computational effort is needed in order to obtain numerically reliable results. Unfortunately, Hatcher et al. used a Morse potential (leading to $S_{1 \mathrm{~V}}^{\mathrm{F}} \approx 2.3 k_{\mathrm{B}}$ ) which yields almost no vacancy relaxation $(-0.02 \Omega)$. Since - as already stressed in ref. [8] - large relaxations alter the result significantly, this potential cannot be considered adequate for the calculation of the formation entropy in $\mathrm{Cu}$.

Following the previous calculations we neglect electronic contributions to $S_{1 \mathrm{~V}}^{\mathrm{F}}$ and consider the vibrational part only. In the quasi-harmonic approximation for temperatures well above the Debye temperature $\Theta$ this gives us

$S_{1 \mathrm{~V}}^{\mathrm{F}}=k_{\mathrm{B}} \sum_{n=1}^{3 N-6} \ln \left(\omega_{n}^{(0)} / \omega_{n}\right), \quad T \gg \Theta$,

where $k_{\mathrm{B}}$ denotes Boltzmann's constant, $N$ the number of atoms in the crystal and $\omega_{n}, \omega_{n}^{(0)}$ the eigenfrequencies of the crystal with or without vacancy. The eigenfrequencies are calculated for infinite crystals with only a limited number $N^{\prime}$ of atoms around the vacancy allowed to vibrate. The presence of relaxations gives 
rise to an additional term due to the volume change $\Delta V_{\mathrm{I}}$ of the finite crystal [6-8], originating from the free surface ("image forces"):

$S_{1 \mathrm{~V}}^{\mathrm{F}}=\lim _{N^{\prime} \rightarrow \infty} S_{\infty}\left(N^{\prime}\right)+\Delta S_{\mathrm{I}}$

with

$$
\begin{aligned}
& S_{\infty}\left(N^{\prime}\right)=k_{\mathrm{B}}\left(\frac{N^{\prime}}{N^{\prime}+1} \sum_{n=1}^{3\left(N^{\prime}+1\right)} \ln \left(\omega_{n, \infty}^{(0)} / \omega_{\mathrm{E}}^{(0)}\right)\right. \\
& \quad-\sum_{n=1}^{3 N^{\prime}} \ln \left(\omega_{n, \infty} / \omega_{\mathrm{E}}^{(0)}\right), \\
& \Delta S_{\mathrm{I}}=\beta K \Delta V_{\mathrm{I}}=\beta K V_{1 \mathrm{~V}}^{\mathrm{rel}}\left(\gamma_{\mathrm{E}}-1\right) / \gamma_{\mathrm{E}} .
\end{aligned}
$$

Here the subscript $\infty$ refers to the infinite crystal; $\omega_{E}^{(0)}$ denotes the Einstein frequency of the perfect crystal, $K$ the bulk modulus, $\beta$ the coefficient of volume thermal expansion and $\gamma_{E}$ the Eshelby factor $[9,10]$. The factor $N^{\prime} /\left(N^{\prime}+1\right)$ takes into account that introducing a vacancy into an unbounded crystal reduces the number of atoms by one.

The expression (4) for the "image term" $\Delta S_{I}$ follows from general thermodynamic arguments and is valid for all temperatures below the melting point. For high temperatures $(T \gg \Theta) \Delta S_{I}$ can also be obtained via the Grüneisen parameter $\gamma_{T}$ for the perfect crystal, which describes the change in the frequency spectrum caused by a homogeneous volume change. The equivalent to eq. (4),

$\Delta S_{\mathrm{I}}=3 k_{\mathrm{B}} \gamma_{\mathrm{T}} \Delta V_{\mathrm{I}} / \Omega$,

with

$\gamma_{\mathrm{T}}=\lim _{N^{\prime} \rightarrow \infty} \frac{1}{3 N^{\prime}} \sum_{n=1}^{3 N^{\prime}}-\frac{\mathrm{d} \ln \omega_{n}^{(0)}}{\mathrm{d} \ln V}$,

will be used to obtain formation entropies entirely deduced from the model potentials without additional experimental information on the anharmonicity of the crystal $[\beta$ in (4)].

$S_{\infty}\left(N^{\prime}\right)$ is expected to be proportional to $1 / N^{\prime}$ for large $N^{\prime}$. For small $N^{\prime}$, however, the discrete character of the lattice dominates and makes an extrapolation $N^{\prime} \rightarrow \infty$ more difficult. An extrapolation using the $1 / N^{\prime}$ behaviour may require $N^{\prime} \geqslant 400$ (corresponding to 15 or more shells of atoms around the vacancy)
[8]. The vast increase of computational requirements associated with the three-body interactions, which range up to third-nearest neighbours, prevented us to reach the $1 / N^{\prime}$ behaviour. The following procedure was adopted: Expression (3) is calculated for the first five shells around the vacancy $\left(N^{\prime}=12,18,42,54\right.$, 78). Atoms with numbers greater than $N^{\prime}$ up to the 40th shell are treated in the Einstein approximation (i.e. for each atom a $3 \times 3$ matrix is diagonalized in order to obtain its Einstein frequency $\omega_{n, \mathrm{E}}$ ). The first five values of
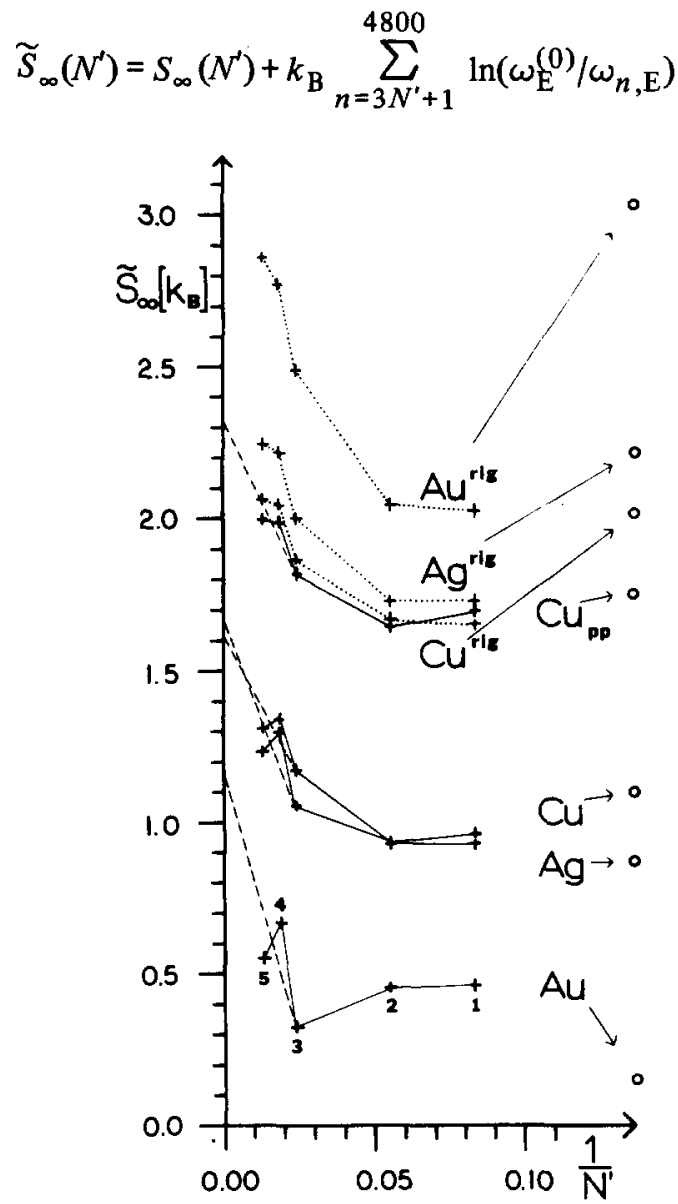

Fig. 1. Results from eq. (7) for the first five shells of atoms around the vacancy obtained with model potentials including three-body interactions $(\mathrm{Cu}, \mathrm{Ag}, \mathrm{Au})$. To illustrate the effect of relaxation the results for a rigid lattice (dotted lines, superscript "rig") and for a pair-potential calculation ( $\mathrm{Cu}_{\mathrm{pp}}$, with very little relaxation) are also shown. For extrapolation to the formation entropy $S_{\infty}(\infty)$ in an infinite crystal (dashed lines) see text. Open circles: Einstein approximation $S_{\infty}\left(N^{+}\right.$ $=0$ ). 
are used for extrapolation to $S_{\infty}(\infty)$.

Fig. 1 shows the five calculated $\widetilde{S}_{\infty}\left(N^{\prime}\right)$ values (connected by straight lines) versus $1 / N^{\prime}$ for different potentials with or without relaxation. $\mathrm{Cu}, \mathrm{Ag}, \mathrm{Au}$ denote the model potentials including three-body interactions which have been used in ref. [1]. The superscript "rig" indicates the result for rigid lattices (i.e. without relaxation). $\mathrm{Cu}_{\mathrm{pp}}$ denotes a pair potential for copper equivalent to the Morse potential used in ref. [8]. For this potential only the case of the relaxed vacancy $\left(V_{1 \mathrm{~V}}=-0.02 \Omega\right)$ is shown.

From fig. 1 the significant effect of relaxation on the formation entropy becomes obvious: The results for $\mathrm{Cu}_{\mathrm{pp}}$ and for $\mathrm{Cu}^{\mathrm{rig}}$ lie close together, whereas those for $\mathrm{Cu}\left(V_{1 \mathrm{~V}}^{\mathrm{rel}}=-0.21 \Omega\right)$ are substantially lower. $\mathrm{Ag}$ is very similar to $\mathrm{Cu}$. For $\mathrm{Au}$ the effect of relaxation is still more pronounced. Qualitatively, these features may be deduced from the Einstein approximation $\widetilde{S}_{\infty}\left(N^{\prime}=0\right)$ indicated by open circles in fig. 1 .

As estimates for $S_{\infty}(\infty)$ and $S_{\infty}^{\text {rig }}(\infty)$ we take the intersections of the dashed straight lines with the vertical axis, which are determined by the 3 -shell value and the point halfway between the 4 - and 5 shell values. The justification for this procedure is that it works in two extreme cases: (i) For $\mathrm{Cu}_{\mathrm{pp}}$ (almost no relaxation) we get $2.3 k_{\mathrm{B}}$, the value reported for the Morse potential in ref. [8]. (ii) For the BornMayer potential treated in ref. [8] (with very large relaxation: $V_{1 \mathrm{~V}}^{\mathrm{rel}}=-0.47 \Omega$ ) we get from fig. 1 in ref. [8] (without the second term in (7)) $1.85 k_{\mathrm{B}}$ instead of the more accurate result $1.75 k_{\mathrm{B}}$. The extrapolated values for $S_{\infty}^{\mathrm{rig}}(\infty)$ and $S_{\infty}(\infty)$ (error probably less than $0.1 k_{\mathrm{B}}$ for $\mathrm{Cu}$ and $\mathrm{Ag}$, and less than $0.2 k_{\mathrm{B}}$ for $\mathrm{Au}$ ) and their difference $\Delta S_{\infty}$ due to the relaxation in the infinite crystal are shown in table 1 . The resulting vacan-

Table 1

Individual contributions (see text) to the vacancy formation entropy $S_{1 \mathrm{~V}}^{\mathrm{F}}$ in units of $k_{\mathrm{B}}$ as determined entirely from the model potentials. $V_{1 \mathrm{~V}}^{\mathrm{rel}}$ is the vacancy relaxation volume already calculated in ref. [1]; $\gamma_{\mathrm{T}}$ is the Grüneisen parameter derived from the model potential.

\begin{tabular}{llllllll}
\hline & $S_{\infty}^{\mathrm{rig}}(\infty)$ & $S_{\infty}(\infty)$ & $\Delta S_{\infty}$ & $\Delta S_{1}$ & $S_{1 \mathrm{~V}}^{\mathrm{F}}$ & $V_{1 \mathrm{~V}}^{\mathrm{rel}}(\Omega)$ & $\gamma_{\mathrm{T}}$ \\
\hline $\mathrm{Cu}$ & 2.40 & 1.62 & -0.78 & -0.58 & 1.04 & -0.21 & 2.80 \\
$\mathrm{Ag}$ & 2.68 & 1.66 & -1.02 & -0.76 & 0.90 & -0.31 & 2.88 \\
$\mathrm{Au}$ & 3.45 & 1.17 & -2.28 & -0.86 & 0.31 & -0.37 & 4.26 \\
\hline
\end{tabular}

cy formation entropy $S_{1 \mathrm{~V}}^{\mathrm{F}}$ in table 1 is obtained by adding $S_{\infty}(\infty)$ and $\Delta S_{I}$ [calculated from eq. (5)]. The Grüneisen parameters $\gamma_{\mathrm{T}}$ derived from the model potentials and the vacancy relaxation volumes $V_{1 \mathrm{~V}}^{\mathrm{F}}$ already given in ref. [1] are also shown.

For comparison we calculate $\Delta S_{\mathrm{I}}$ from (4). All quantities in (4) are temperature dependent, but because of positive (for $\beta$ ) and negative (for $K$ and $\left(\gamma_{\mathrm{E}}\right.$ $\left.-1) / \gamma_{E}\right)$ temperature coefficients the prefactor of $V_{1 \mathrm{~V}}^{\mathrm{rel}}$ in (4) varies only little between $300 \mathrm{~K}$ and the melting point. We evaluate (4) with experimental values of $\beta$ and $c_{i j}$ at $800 \mathrm{~K}$ assuming for simplicity that $V_{1 \mathrm{~V}}^{\mathrm{rel}} / \Omega(T)$ is independent of temperature. The image contributions $\Delta S_{\mathrm{I}}^{\text {corr }}$ obtained this way are smaller by factors of $1.44,1.21$, and 1.38 for $\mathrm{Cu}$, $\mathrm{Ag}$, and $\mathrm{Au}$, respectively, leading to higher values of $S_{1 \mathrm{~V}}^{\mathrm{F}}$ (first and second column of table 2). Since (4) and (5) are equivalent we conclude that the Grüneisen parameters derived from the model potentials are too large by these factors, i.e. the anharmonicity changing the frequencies of the crystal in response to a homogeneous volume change is too strong. The same effect on the frequencies can be expected for an inhomogeneous distortion like the relaxation in the infinite crystal, and consequently we have to correct the contribution $\Delta S_{\infty}$, too. For simplicity we use the same factors already used for $\Delta S_{\mathrm{I}}$. The reduced values $\Delta S_{\infty}^{c o r r}$ cause a further increase in the formation entropy (last two columns of table 2).

The results for $S_{1 \mathrm{~F}}^{\mathrm{F}}$ in columns two and four of table 2 can be regarded as lower and upper bounds, respectively (subject to the extrapolation errors mentioned above). Column 2 gives lower bounds because only $\Delta S_{\mathrm{I}}$ is corrected and $\Delta S_{\infty}$ is still overestimated by the too strong anharmonicity of the potentials. Column 4 gives upper bounds because the absolute

Table 2

Corrected values for $\Delta S_{\mathrm{I}}$ and $\Delta S_{\infty}$ and final estimates for $S_{1 \mathrm{~V}}^{\mathrm{F}}$ in units of $k_{\mathrm{B}}$ (1.b. = lower bound, u.b. = upper bound).

\begin{tabular}{lllll}
\hline & $\Delta S_{\mathrm{I}}^{\text {corr }}$ & $S_{\mathrm{IV}}^{\mathrm{F}}$ & $\Delta S_{\infty}^{\text {corr }}$ & $S_{\mathrm{1V}}^{\mathrm{F}}$ \\
& & $1 . \mathrm{b}$. & & $\mathrm{u.b.}$ \\
\hline $\mathrm{Cu}$ & -0.40 & 1.22 & -0.54 & 1.46 \\
$\mathrm{Ag}$ & -0.63 & 1.03 & -0.84 & 1.21 \\
$\mathrm{Au}$ & -0.62 & 0.55 & -1.65 & 1.18 \\
\hline
\end{tabular}


values of $\Delta S_{\infty}^{\text {corr }}$, which cannot be justified rigorously, are very likely underestimated for the following reason: The image term $\Delta S_{\mathrm{I}}$ is related to a homogeneous relaxation of the lattice and hence to the bulk modulus and its pressure derivative. Conversely, $\Delta S_{\infty}$ is related to an inhomogeneous relaxation and therefore essentially to the shear moduli and their pressure derivatives. Compared to experimental values the Grüneisen parameters and pressure derivatives of the bulk moduli derived from the potentials show very similar deviations, whereas the pressure derivatives of the shear moduli are in significantly better agreement with experimental values, although too high as well. Hence, $\Delta S_{\infty}$ should really be corrected by somewhat smaller factors than $\Delta S_{\mathrm{I}}$, leading to lower values of $S_{1 \mathrm{~V}}^{\mathrm{F}}$.

We arive at the conclusion that for calculations of vacancy formation entropies with an accuracy of about $0.1 k_{\mathrm{B}}$ high-quality model potentials are required. The model potentials should be able to reproduce the vacancy relaxation volume as well as - especially in the case of large relaxations - the anharmonic properties of the crystal like Grüneisen parameter and pressure derivatives of the elastic constants. The Morse potential used by Hatcher et al. [8] does not even meet the first requirement and has to be considered inappropriate for calculating $s_{1 \mathrm{~V}}^{\mathrm{F}}$ for $\mathrm{Cu}$. Their result $\left(2.3 k_{\mathrm{B}}\right)$ comes out too large. Including three-body interactions in the model potential makes it possible to meet the first requirement. Nevertheless, the model potentials used in the present work are not completely satisfactory with respect to anharmonic properties. In particular, the model potential for Au needs to be improved.

For $\mathrm{Cu}$ and $\mathrm{Ag}$ we have calculated lower and upper bounds for $S_{1 \mathrm{~V}}^{\mathrm{F}}$, which differ only by approximately $0.2 k_{\mathrm{B}} \neq 1$. Taking into account an extrapolation error of $\pm 0.1 k_{\mathrm{B}}$ we expect experimental values within these limits. Additional uncertainties due to the neglected temperature dependence of the eigenfrequencies are presumably small.

¥1 If we use the $\mathrm{Cu}$ potential with van der Waals exponent $n_{\mathrm{vdW}}=5$ which yields a vacancy formation energy of $1.32 \mathrm{eV}$ (instead of $1.05 \mathrm{eV}$ with $n_{\mathrm{vdW}}=6$ ) but also $V_{1 \mathrm{~V}}^{\text {rel }}$ $=-0.21 \Omega[1]$, we obtain very similar results (e.g., $S_{1 \mathrm{~V}}^{\mathrm{F}}$ $=1.29 k_{\mathrm{B}}$ and $1.49 k_{\mathrm{B}}$ for lower and upper bound, respectively).

\section{References}

[1] R. Bauer, W. Maysenhölder and A. Seeger, Phys. Lett. 90A (1982) 55.

[2] R. Bauer, unpublished.

[3] B.M. Axilrod and E. Teller, J. Chem. Phys. 11 (1941) 299.

[4] H.-G. Haubold and D. Martinsen, J. Nucl. Mater. $69+70$ (1978) 644.

[5] P.H. Dederichs, C. Lehmann, H.R. Schober, A. Scholz and R. Zeller, J. Nucl. Mater. $69+70$ (1978) 176.

[6] H.B. Huntington, G.A. Shirn and E.S. Wajda, Phys. Rev. 99 (1955) 1085.

[7] G. Schottky, A. Seeger and G. Schmid, Phys. Stat. Sol. 4 (1965) 439.

[8] R.D. Hatcher, R. Zeller and P.H. Dederichs, Phys. Rev. B19 (1979) 5083.

[9] J.D. Eshelby, J. Appl. Phys. 25 (1954) 255.

[10] W. Maysenhölder, Phys. Lett. 100A (1984) 289. 